\title{
Vascular Targeted Radioimmunotherapy for the Treatment of Glioblastoma
}

\author{
Katja Behling ${ }^{1}$, William F. Maguire ${ }^{2}$, José Carlos López Puebla ${ }^{1}$, Shanna R. Sprinkle ${ }^{1}$, Alessandro Ruggiero ${ }^{1}$, \\ Joseph O’Donoghue ${ }^{3}$, Philip H. Gutin ${ }^{4,5}$, David A. Scheinberg ${ }^{2,6}$, and Michael R. McDevitt ${ }^{1,7}$ \\ ${ }^{1}$ Department of Radiology, Memorial Sloan Kettering Cancer Center, New York, New York; ${ }^{2}$ Department of Molecular Pharmacology, \\ Memorial Sloan Kettering Cancer Center, New York, New York; ${ }^{3}$ Department of Medical Physics, Memorial Sloan Kettering Cancer \\ Center, New York, New York; ${ }^{4}$ Department of Neurosurgery, Memorial Sloan Kettering Cancer Center, New York, New York; \\ ${ }^{5}$ Department of Neurological Surgery, Weill Cornell Medical College, New York, New York; ${ }^{6}$ Department of Pharmacology, Weill \\ Cornell Medical College, New York, New York; and ${ }^{7}$ Department of Medicine, Weill Cornell Medical College, New York, New York
}

Glioblastoma is characterized by an aggressive and aberrant vascular network that promotes tumor progression and hinders effective treatment; the median survival is 16 mo despite standardof-care therapies. There is a need to improve therapeutic options for this disease. We hypothesized that antibody targeting of the vascular endothelium of glioblastoma with cytotoxic short-range, high-energy a-particles would be an effective therapeutic approach. Methods: E4G10, an antibody directed at an epitope of monomeric vascular endothelium cadherin that is expressed in tumor neovasculature and on endothelial progenitor cells in the bone marrow, was labeled with a-particle-emitting ${ }^{225} \mathrm{Ac}$. Pharmacokinetic studies investigated the tissue distribution and blood clearance of the ${ }^{225} \mathrm{Ac}-\mathrm{E} 4 \mathrm{G} 10$ radioimmunoconstruct in a transgenic Nestin-tumor virus A (Ntva) mouse model of high-grade glioblastoma. Histologic analysis was used to demonstrate local therapeutic effects in treated brain tumor sections. Radioimmunotherapy with ${ }^{225}$ Ac-E4G10 was performed in Ntva mice to assess overall survival alone and in combination with temozolomide, the standard-of-care chemotherapeutic agent. Results: ${ }^{225} \mathrm{Ac}-\mathrm{E} 4 \mathrm{G} 10$ was found to accumulate in tissues expressing the target antigen. Antivascular a-particle therapy of glioblastoma in the transgenic Ntva model resulted in significantly improved survival compared with controls and potent control of tumor growth. Adding the chemotherapeutic temozolomide to the treatment increased survival to $30 \mathrm{~d}$ (vs. $9 \mathrm{~d}$ for vehicle-treated animals). Histologic analyses showed a remodeled glioblastoma vascular microenvironment. Conclusion: Targeted a-particle antivascular therapy is shown for the first time to be effective in increasing overall survival in a solid tumor in a clinically relevant transgenic glioblastoma mouse model.

Key Words: radioimmunotherapy (RIT); ${ }^{225} \mathrm{Ac}$; glioblastoma (GBM); vascular endothelium (VE)

J Nucl Med 2016; 57:1576-1582

DOI: 10.2967/jnumed.115.171371

Received Dec. 15, 2015; revision accepted Mar. 23, 2016.

For correspondence or reprints contact either of the following:

Katja Behling, Department of Radiology, Memorial Sloan Kettering Cancer

Center, 1275 York Ave., P.O. Box 231, New York, NY 10065.

E-mail: behlingk@mskcc.org

Michael R. McDevitt, Department of Radiology, Memorial Sloan Kettering

Cancer Center, 1275 York Ave., P.O. Box 231, New York, NY 10065.

E-mail: m-mcdevitt@ski.mskcc.org

Published online Apr. 28, 2016.

COPYRIGHT (c) 2016 by the Society of Nuclear Medicine and Molecular Imaging, Inc.
G ioblastoma is a malignant astrocytoma and one of the most rapidly fatal and incurable cancers (1). These aggressive tumors are highly invasive, and their cellular processes can often extend several centimeters away from the primary lesion. Standard intervention is surgical resection followed by field radiotherapy (total dose of up to $60 \mathrm{~Gy}$ ). Chemotherapy improves the outcome, but despite the aggressive combination of surgery, radiotherapy, and chemotherapy, the median survival is only 16 mo (2). Clinical outcomes for patients with glioblastoma have remained the same for many years despite numerous attempts to improve the standard of care. The probability of 2-y survival is less than $30 \%$ with optimal therapy (3). There is a need to provide better therapeutic options for this disease.

Glioblastoma is among the most vascularized tumors (4), making it an ideal target for antivascular therapies (5). Bevacizumab, the first clinically validated antiangiogenic therapeutic for glioblastoma, inhibits vascular endothelial growth factor A signaling, which has a central role in blood vessel growth (6). However, systemic toxicity has been an important issue when treating with bevacizumab (7), as well as observed resistance to the treatment (8) and only minimally improved survival benefit.

Damaging the tumor's vascular endothelium (VE) is another antivascular therapeutic strategy $(9,10)$. $\alpha$-particles are charged helium nuclei that travel only several cell diameters $(40-80 \mu \mathrm{m})$. Individual $\alpha$-particles deposit 5-8 MeV of energy over a short, densely ionizing track, causing cell death. This is $10-100$ times more energy than is released in a $\beta$-particle emission typically used in radioimmunotherapy. $\alpha$-particles are lethal when emitted in the vicinity of a target cell but spare normal bystander tissue outside their short range (11-13). This characteristic particle energy and geometry offer clear advantages over other known forms of radiation as a means of precision cell killing, particularly in the brain. ${ }^{225} \mathrm{Ac}$ is an $\alpha$-particleemitting radionuclide (half-life, $10.0 \mathrm{~d}$ ) that, along with its daughters, releases $4 \alpha$-particle emissions, each individually lethal to cells in its path. This ${ }^{225} \mathrm{Ac}$-antibody drug strategy has been described as an atomic nanogenerator for in vivo delivery of $\alpha$-particles to tumor (12).

$\alpha$-particle-emitting radionuclides are among the most potent cytotoxic agents known, and the approval of Xofigo (223 Ra-chloride; Bayer Healthcare) by the Food and Drug Administration demonstrated the effectiveness and safety of targeted $\alpha$-particle therapy (14). We translated ${ }^{225}$ Ac-lintuzumab into clinical trials against leukemia (15). To date, we have completed a crucial phase I study of 
${ }^{225}$ Ac-lintuzumab, demonstrating safety and biologic activity, leading to a multicenter phase I/II trial to determine the maximum tolerated dose, toxicity, and efficacy of fractionated doses of ${ }^{225}$ Ac-lintuzumab in combination with low-dose cytarabine. We brought this knowledge to bear on the development of the VEtargeted antibody E4G10, a monoclonal antibody that binds to an epitope of monomeric VE-cadherin that is expressed by angiogenic VE $(16,17)$ and bone marrow-derived endothelial progenitor cells (18). Importantly, E4G10 binds to an epitope present only on monomeric VE-cadherin but not the homodimeric form found in established resting vasculature, thus conferring specificity for tumor vessels and endothelial progenitor cells.

Here, we report the first, to our knowledge, preclinical investigation of cytotoxic $\alpha$-particle irradiation as antivascular treatment of highgrade glioblastoma in a transgenic mouse model of rapidly progressing disease. We first investigate the biodistribution of the ${ }^{225} \mathrm{Ac}-\mathrm{E} 4 \mathrm{G} 10$ radioimmunoconstruct in naïve and glioblastoma-bearing mice and show local histologic response to the treatment in brain tumor tissue. In survival studies, we explore the therapeutic ability of radioimmunotherapy with ${ }^{225}$ Ac-E4G10 in Nestin-tumor virus A (Ntva) mice as stand-alone therapy and in combination with the standard-of-care chemotherapeutic temozolomide. Targeted $\alpha$-particle antivascular therapy is investigated for the first time in a solid tumor in a relevant transgenic glioblastoma mouse model.

\section{MATERIALS AND METHODS}

\section{Radiochemistry}

${ }^{225} \mathrm{Ac}$ (ORNL) was conjugated to the anti-VE-cadherin IgG2a rat antibody E4G10 (Eli Lilly and Co.) or an isotype-matched control antibody (IgG2a, anti-Keyhole Limpet Haemocyanin; R\&D Systems) and purified and analyzed for radiolabeling quality as described previously $(19,20)$. The purified radioimmunoconstructs were formulated in $1 \%$ human serum albumin (HSA; Swiss Red Cross) and $0.9 \%$ sodium chloride (Normal Saline Solution; Abbott Laboratories) for intravenous injection.

\section{Glioblastoma Animal Model}

The Ntva mouse (Ink4a-Arf ${ }^{-1-} ; \mathrm{FVB} / \mathrm{N}, \mathrm{C} 57 \mathrm{BL6}$, and BALB/C background) with the retroviral RCAS (replication-competent avian sarcoma-leukosis virus) system was previously described (21) and used for all studies in vivo. The RCAS vector carries the tumor-driver human platelet-derived growth factor B and is overexpressed by chicken DF-1 cells. Tumor induction was performed when Ntva mice were $4-10$ wk old by stereotactic delivery of $2 \times 10^{5}$ chicken DF- 1 cells $(1 \mu \mathrm{L})$ intracranially at $1-\mathrm{mm}$ caudal and $1.5-\mathrm{mm}$ lateral from bregma into the right frontal cortex (injection depth, $2 \mathrm{~mm}$ ).

\section{Experimental Design for Studies In Vivo}

Tumor growth and progression of glioblastoma-induced Ntva mice was assessed by performing T2 MRI 5 wk after induction. Animals were subsequently placed into treatment groups with a distribution of tumor sizes and treated with either $0.1 \mathrm{~mL}$ of radioimmunocontruct or injection vehicle, respectively, via retroorbital venous plexus. Animals were monitored for survival or sacrificed $10 \mathrm{~d}$ after the treatment and tissues harvested for analysis.

\section{T2 MRI}

Mice were MR-imaged to quantify initial tumor volumes for therapeutic studies and to assess treatment responses with a custom-made (inner diameter, $32 \mathrm{~mm}$ ) quadrature birdcage body resonator (Stark Contrast MRI Research) while anesthetized under 1.5\% isoflurane. All images were acquired on a Bruker USR 4.7-T scanner (Bruker Biospin MRI Inc.). The mouse head was imaged in coronal orientation using a T2-weighted fast spin-echo rapid imaging with refocused echo (RARE) sequence (repetition time, $3.5 \mathrm{~s}$; echo time, $50 \mathrm{~ms}$; RARE factor, 8; number of excitations, 24; field of view, $3 \times 2 \mathrm{~cm}$; slice thickness, 0.7 or $1 \mathrm{~mm}$; in-plane resolution, $117 \times 156 \mu \mathrm{m}$ ).

\section{Pharmacokinetic Profile of Radioimmunoconstructs}

Twenty-four Ntva mice with glioblastoma were MR-imaged for tumor growth and tumor sizes and placed in 8 groups $(n=3)$; 40 naïve Ntva mice were arranged into 8 groups $(n=5)$. Each animal received a single intravenous dose of $11.1 \mathrm{kBq}(300 \mathrm{nCi})$ of ${ }^{225} \mathrm{Ac}-\mathrm{E} 4 \mathrm{G} 10$ or ${ }^{225} \mathrm{Ac}$-isotype. Mice were euthanized at 4, 12, 36, and $240 \mathrm{~h}$, and tissue samples were harvested, weighed, and counted in a $\gamma$-counter using a $360-$ to $480-\mathrm{keV}$ window at secular equilibrium. Aliquots $(20 \mu \mathrm{L})$ of the injected doses were used as decay-correction standards. The percentage injected doses per gram of tissue weight were calculated and plotted as means. Statistical analysis of data was performed using Prism software (GraphPad Software Inc.).

\section{Absorbed Dose Estimates}

The percentage injected dose per gram of tissue weight values were converted to percentage of injected activity (\%IA) per gram of tissue weight. Thereafter, the areas under the activity concentration-time curves were estimated by trapezoidal integration with the contribution of the terminal portion calculated by extrapolation from the 240 -h value using the faster of apparent terminal clearance rate or physical decay. Subsequently, absorbed doses, D (Gy/MBq), were calculated from the area under the curve $(\% \mathrm{IA} \mathrm{h} / \mathrm{g})$ values according to $\mathrm{D}=10 \times$ area under the curve $\times \Delta$, where $\Delta$ is the equilibrium dose constant for the total decay of ${ }^{225} \mathrm{Ac}$. The value used for $\Delta\left(1.43 \times 10^{-2} \mathrm{~J} / \mathrm{MBq} \mathrm{h}\right)$ includes the contributions of all the radioactive progeny of ${ }^{225} \mathrm{Ac}$ and thus assumes no translocation of any progeny from the site of the original ${ }^{225} \mathrm{Ac}$ decay.

\section{Histologic Analysis}

Glioblastoma-bearing Ntva mice $(n=12)$ were placed into the following groups with an even distribution of tumor sizes: ${ }^{225} \mathrm{Ac}-\mathrm{E} 4 \mathrm{G} 10$ $(n=4),{ }^{225}$ Ac-isotype $(n=4)$, and $1 \%$ HSA vehicle $(n=4)$. Anesthetized ( $1.5 \%$ isoflurane) mice received a single $7.4-\mathrm{kBq}(200 \mathrm{nCi})$ dose $(0.1 \mathrm{~mL})$ via retroorbital venous plexus of ${ }^{225} \mathrm{Ac}-\mathrm{E} 4 \mathrm{G} 10,{ }^{225} \mathrm{Ac}$-isotype control, or vehicle at day 0 . Ten days after treatment, the tumor was harvested and analyzed. Briefly, mice were sacrificed and glioblastoma and brain were excised and fixed in $4 \%$ paraformaldehyde/phosphate-buffered saline for $2 \mathrm{~d}$. Fixed tissue was paraffin-embedded and cut into 5 - $\mu \mathrm{m}$ sections. Sections were stained with hematoxylin and eosin or anti-CD31antibody for endothelium, respectively. Sections were scanned with the Mirax Digital Slide Scanner using a 20× lens (Carl Zeiss Microimaging). Analysis was performed on whole tumor sections with Pannoramic Viewer software (3DHISTECH).

\section{Survival Studies}

Therapy was conducted for all survival studies after the initial MR imaging $5 \mathrm{wk}$ after induction. Tumor induction with the RCAS system resulted in a normal distribution of tumor sizes and disease progression in tumor-induced mice $(60 \%-80 \%$ of tumors small/medium sized; $10 \%-20 \%$ very small/not visible in the initial MRI; $10 \%-20 \%$ were large tumors occupying up to $20 \%$ of cranial space). Mice with large tumor sizes exhibited glioblastoma symptoms such as hydrocephalus or hunched posture and were excluded from entering studies (as well as animals exhibiting very small/not-yet-visible tumors). However, these late-stage animals entered survival study II to investigate therapy results in a late-stage disease scenario. Survival outcomes were scored and plotted using Kaplan-Meier survival analysis in Prism software.

\section{Survival Study I}

Mice were separated into a ${ }^{225} \mathrm{Ac}-\mathrm{E} 4 \mathrm{G} 10$ treatment group $(n=9)$ and a $1 \%$ HSA (vehicle) group $(n=8)$ and treated with $0.46 \mathrm{kBq}(12.5 \mathrm{nCi})$ of ${ }^{225} \mathrm{Ac}-\mathrm{E} 4 \mathrm{G} 10 / \mathrm{g}$ of body mass (7.4-11.1 kBq [200-300 nCi]/mouse). 


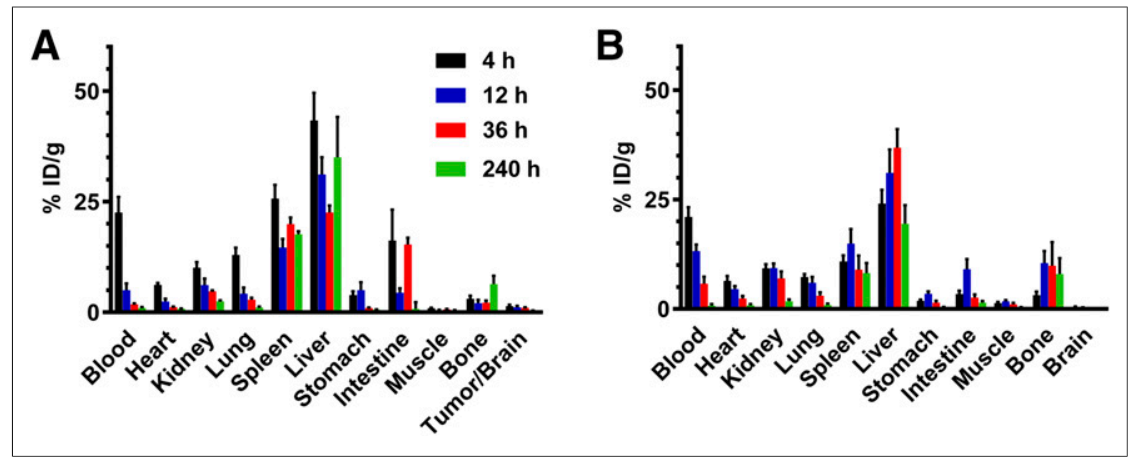

FIGURE 1. Tissue biodistribution of ${ }^{225} \mathrm{Ac}-\mathrm{E} 4 \mathrm{G} 10$ in glioblastoma tumor-bearing mice $(\mathrm{A})$ and in naïve mice (B). Shown is tissue uptake in percentage injected dose per gram of tissue weight $(\% \mathrm{ID} / \mathrm{g})$ at different time points. Reported values are mean \pm SEM.

Tumor growth was followed in 4 representative mice per group using T2 MRI to measure tumor volume at baseline (day 0; 5 wk after tumor induction) and $10 \mathrm{~d}$ later.

\section{Survival Study II}

Mice treated in survival study II exhibited advanced glioblastoma with symptoms of hydrocephalus and hunched posture. Mice were separated into a specific ${ }^{225} \mathrm{Ac}-\mathrm{E} 4 \mathrm{G} 10$ treatment group $(n=4)$, a control ${ }^{225} \mathrm{Ac}-$ isotype group $(n=3)$, and a $1 \%$ HSA vehicle-only control group $(n=6)$. Mice were treated $5 \mathrm{wk}$ after tumor induction with $0.46 \mathrm{kBq}(12.5 \mathrm{nCi}) / \mathrm{g}$ body mass $(7.4-11.1 \mathrm{kBq}[200-300 \mathrm{nCi}] /$ mouse) or with the $1 \% \mathrm{HSA}$ injection vehicle.

\section{Survival Study III}

Animals in the ${ }^{225} \mathrm{Ac}-\mathrm{E} 4 \mathrm{G} 10$ plus temozolomide group $(n=10)$ received $0.46 \mathrm{kBq}(12.5 \mathrm{nCi}) / \mathrm{g}$ body mass $(7.4-11.1 \mathrm{kBq}[200-300 \mathrm{nCi}] /$ outcomes.

\section{Study Approval}

\section{RESULTS} Mantel-Cox) and Mantel-Haenszel tests were used to compare survival

All animal experiments were done in accordance with the National Institutes of Health (22) guide for the care and use of laboratory animals and approved by the Institutional Animal Care and Use Committee of Memorial Sloan Kettering Cancer Institute.

\section{Radioimmunotherapeutic Drug ${ }^{225} \mathrm{Ac}-\mathrm{E} 4 \mathrm{G} 10$ and Ntva Animal Model}

The transgenic Ntva mouse modeled the platelet-derived growth factor-driven proneural glioblastoma subtype of human disease and was used to evaluate ${ }^{225} \mathrm{Ac}-\mathrm{E} 4 \mathrm{G} 10$ in therapeutic studies (21). Radiolabeling results were similar for the specific antibody E4G10 and the isotype control antibody used in therapeutic and mechanistic studies. The ${ }^{225}$ Ac-E4G10 constructs were $97.6 \% \pm 2.4 \%$ radiochemically pure (mean $\pm \mathrm{SD} ; n=16$ ) and had specific activities of $7.03 \pm 3.70 \mathrm{GBq}(0.19 \pm 0.10$ $\mathrm{Ci}) / \mathrm{g}$. The ${ }^{225} \mathrm{Ac}$-isotype was $98.7 \% \pm$ $0.84 \%(n=10)$ radiochemically pure and had specific activities of $4.81 \pm 2.96 \mathrm{GBq}$ $(0.13 \pm 0.08 \mathrm{Ci}) / \mathrm{g}$.

\section{Pharmacokinetic Profile of ${ }^{225}$ Ac-E4G10 in Naïve and Glioblastoma Ntva Mice}

The biodistribution of ${ }^{225} \mathrm{Ac}-\mathrm{E} 4 \mathrm{G} 10$ and ${ }^{225}$ Ac-isotype was profiled in Ntva mice with and without glioblastoma as a function of time (Fig. 1; Supplemental Fig. 1 [supplemental materials are available at http://jnm. snmjournals.org]). ${ }^{225}$ Ac-E4G10 cleared from the blood in glioblastoma mice within the first $2 \mathrm{~d}$ of treatment (Figs. 1A and 2A) whereas naïve Ntva mice cleared the drug more slowly from the blood (Fig. 2B), presumably because in the mice with tumors there were sinks for the drug to accumulate. The low activity accumulated in tumor can be attributed to the small number of monomeric VE-cadherin ${ }^{+}$ cells being targeted in the mouse (the tumor 


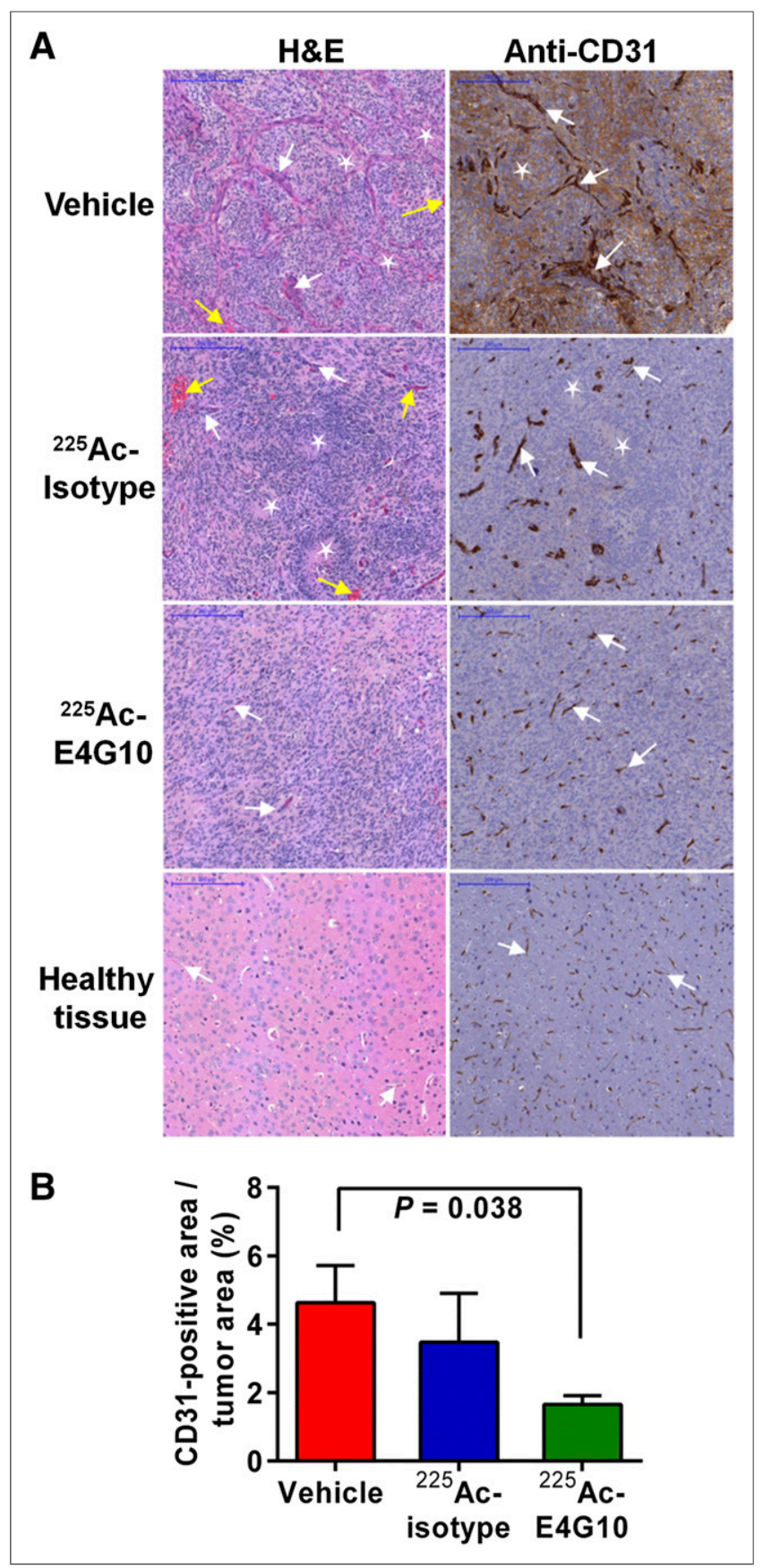

FIGURE 3. Histologic response of glioblastoma to targeted ${ }^{225} \mathrm{AC}-$ E4G10 therapy in Ntva mice. (A) Representative sections from treated glioblastoma Ntva mice stained with hematoxylin and eosin (H\&E) or anti-CD31 antibody for blood vessels. Healthy brain tissue is included for comparison. Blood vessels are indicated by white arrows, necrosis is indicated with asterisk, and hemorrhage is indicated with yellow arrow. Scale bars are $200 \mu \mathrm{m}$ in all panels. (B) Quantification of vascularity of whole anti-CD31-stained tumor sections of treated glioblastoma mice $(n=4)$. Values are mean \pm SEM.

cells are not targeted directly with this drug). Plotting the activity measured in the tumor and bone (which includes the red marrow) normalized to blood activity (Figs. $2 \mathrm{C}$ and $2 \mathrm{D}$ ) reveals a timedependent enrichment of the labeled antibody in glioblastoma/brain and bone versus blood. Monomeric VE-cadherin is expressed both in the glioblastoma vasculature and in the endothelial progenitor cells in the red marrow. The glioblastoma and bone data normalized to blood activity show less accumulation of the isotype control drug construct. The ${ }^{225}$ Ac-isotype control antibody exhibited a prolonged blood half-life compared with ${ }^{225}$ Ac-E4G10 (Supplemental Fig. 1; Fig. $2 \mathrm{~A}$ ): $11.5 \%$ in the blood at $36 \mathrm{~h}$ and $5.2 \%$ at $240 \mathrm{~h}$ (vs. $1.8 \%$ at $36 \mathrm{~h}$ in the ${ }^{225} \mathrm{Ac}-\mathrm{E} 4 \mathrm{G} 10$ group).

\section{Absorbed Dose Estimates of ${ }^{225}$ Ac-E4G10 in Naïve and Glioblastoma Ntva Mice}

We performed dosimetry calculations from the biodistribution data to estimate the tumor absorbed dose and to estimate doses to nontargeted tissues (Supplemental Table 1). The estimated absorbed dose of ${ }^{225} \mathrm{Ac}-\mathrm{E} 4 \mathrm{G} 10$ in glioblastoma-bearing mice for tumor/brain was found to be $24.4 \pm 4.8 \mathrm{~Gy} / \mathrm{MBq}$. In comparison, the estimated dose to healthy brain was $3.1 \pm 0.8 \mathrm{~Gy} / \mathrm{MBq}$. Hence, the estimated absorbed dose to tumor tissue was 7.7 times higher than that to healthy brain tissue $(P=0.016)$.

\section{Histologic Response to ${ }^{225}$ Ac-E4G10 Treatment}

The gross architecture of the glioblastoma vascular endothelial microenvironment changed as a result of ${ }^{225}$ Ac-E4G10 therapy. Glioblastoma tissue sections were examined $10 \mathrm{~d}$ after therapy with hematoxylin and eosin staining and anti-CD31 endothelial cell marker (Fig. 3). Vehicle-treated tumors showed characteristic hematoxylin and eosin morphology for high-grade glioblastoma. Massive branches of vasculature are visible, and the cellularity is increased. The tumor vasculature is morphologically distinct from healthy brain. Vessels in glioblastoma spread without organization, are tortuous, and show massive extensions in all directions. They are leaky (edematous), dilated, irregular in shape, and have diameters up to $100 \mu \mathrm{m}$. A single dose of ${ }^{225} \mathrm{Ac}-\mathrm{E} 4 \mathrm{G} 10$ leads to significant glioblastoma VE remodeling. These mice exhibited structural vascular changes with features analogous to healthy brain in which vessels are significantly smaller in size and diameter compared with controls. There are more necrotic regions noted in the vehicle and isotype control antibody groups as well as a greater CD31 density $(P=0.038)$ than in vehicle-treated controls (Fig. 3B). The intermediate changes in CD31 arising from ${ }^{225} \mathrm{Ac}$-isotype were attributed to nonspecific irradiation from the activity that persisted in blood (Figs. 2C, 3A, and $3 \mathrm{~B})$.

\section{Vascular Targeting ${ }^{225} \mathrm{Ac}-\mathrm{E} 4 \mathrm{G} 10$ Controls Tumor Growth}

Tumors in animals treated with ${ }^{225}$ Ac-E4G10 in survival study I were significantly smaller than tumors in the vehicle-treated group $(P=0.017$, Figs. 4A and 4B) $10 \mathrm{~d}$ after therapy. T2 MRI of 4 representative animals per group of animals from survival study I showed that baseline tumor volumes at day 0 of treatment $(5 \mathrm{wk}$ after induction) were $31.5 \pm 13.0$ and $10.0 \pm 4.9 \mathrm{~mm}^{3}$ in the vehicle and E4G10 groups, respectively. On day 10, animals in the vehicle-treated group had mean tumor volumes of $171.7 \pm$ 28.6 versus $42.7 \pm 26.9 \mathrm{~mm}^{3}$ for ${ }^{225} \mathrm{Ac}-\mathrm{E} 4 \mathrm{G} 10$-treated animals. At the start of therapy, approximately $4 \%$ (on average) of the cranial space was occupied by glioblastoma, and by day 10 the untreated control mice had tumor volumes occupying approximately $35 \%$ on average of the cranial space.

\section{${ }^{225}$ AC-E4G10 Radioimmunotherapy of Glioblastoma Vasculature Significantly Increases Survival}

In survival study I (Fig. 5A), we asked whether ${ }^{225} \mathrm{Ac}-\mathrm{E} 4 \mathrm{G} 10$ treatment improves overall survival of glioblastoma-bearing Ntva mice and whether the radioimmunoconstruct was active toward 


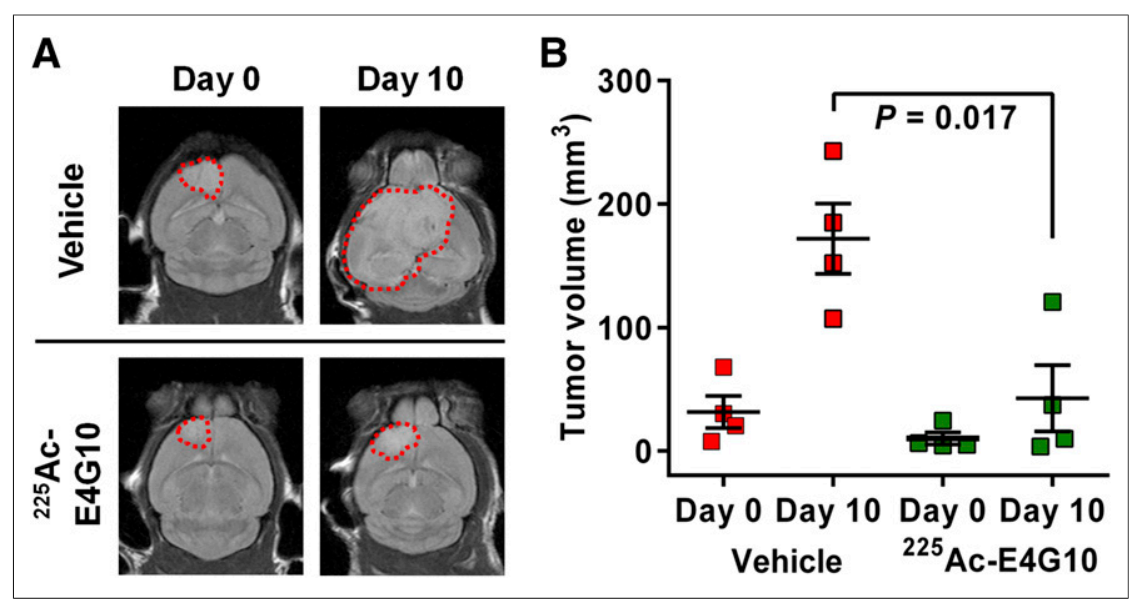

FIGURE 4. Tumor sizes of glioblastoma-bearing animals in survival study I before and $10 \mathrm{~d}$ after treatment with either specific treatment ${ }^{225} \mathrm{Ac}-\mathrm{E} 4 \mathrm{G} 10$ or injection vehicle accessed by MRI. Shown is T2 MR scan in coronal orientation of 1 representative animal of each group in A. Tumor margins are displayed as dashed red lines. (B) Mean tumor volumes at day 0 and day 10 of vehicle-treated $(n=4)$ versus ${ }^{225}$ Ac-E4G10-treated $(n=4)$ animals. Reported values are mean \pm SEM.

inhibiting glioblastoma growth (Fig. 4B). Treatment with ${ }^{225}$ AcE4G10 was found to significantly prolong median survival compared with controls. The median survival in the ${ }^{225}$ Ac-E4G10 group was $21 \mathrm{~d}$ compared with $9 \mathrm{~d}$ in the vehicle group $(P=$ 0.0002, Fig. 5A). Survival study II was conducted to demonstrate efficacy of treatment in animals with advanced disease symptoms and to show specificity of ${ }^{225} \mathrm{Ac}-\mathrm{E} 4 \mathrm{G} 10$ for increasing survival by comparison to the ${ }^{225} \mathrm{Ac}$-labeled isotype control antibody or vehicle (Fig. 5B). ${ }^{225}$ Ac-E4G10 treatment prolonged median survival significantly compared with controls. The 3 groups had median survival values of $11 \mathrm{~d}$ for ${ }^{225} \mathrm{Ac}-\mathrm{E} 4 \mathrm{G} 10$ versus $7 \mathrm{~d}$ for ${ }^{225} \mathrm{Ac}$ isotype versus $5 \mathrm{~d}$ for the vehicle group, respectively. Survival study III (Fig. 5C) compared the efficacy of combined ${ }^{225}$ AcE4G10 radioimmunotherapy and temozolomide chemotherapy in groups of mice with glioblastoma. The combinational therapy prolonged the median survival of Ntva mice to $30 \mathrm{~d}$ in the ${ }^{225} \mathrm{Ac}-\mathrm{E} 4 \mathrm{G} 10$ plus temozolomide group versus $26 \mathrm{~d}$ in the temozolomide-only group. The survival benefit in the combination group was not statistically different than temozolomide alone $(P=0.128)$. However, the Kaplan-Meier plot of study III shows an extended survival tail, reflecting the ${ }^{225}$ Ac-E4G10 plus temozolomide therapeutic benefit. Forty percent of animals in the combination therapy group were still alive on day 32 compared with $0 \%$ in the temozolomide-only group. The hazard ratio was found to be 0.4973 ( ${ }^{225}$ Ac-E4G10 plus temozolomide/temozolomide), expressing the survival benefit for animals in the combined therapy group.

\section{DISCUSSION}

Glioblastoma is one of the most aggressive and vascularized tumors and therefore a strategic objective for the potent antivascular ${ }^{225}$ Ac-E4G10 drug. We have reported the therapeutic effectiveness of ${ }^{225}$ Ac-E4G10 directed against xenografted carcinoma VE and bone marrow-endothelial progenitor cells $(18,23-25)$ and now directed this drug against the VE of glioblastoma. We wanted to explore the therapeutic potential of ${ }^{225} \mathrm{Ac}-\mathrm{E} 4 \mathrm{G} 10$ arising from changes produced by $\alpha$-irradiation in the glioblastoma vasculature. The glioblastoma blood-brain barrier presents a unique vascular microenvironment compared with the other carcinoma models that we have studied. The Ntva model is a genetically engineered mouse that recapitulates the genetics and histology of the human glioblastoma $(21,26)$ and provided a relevant orthotopic model for our investigations. Moreover, Ntva mice are not immunodeficient, which permits the interpretation of therapy outcomes under immunocompetent background.

$\alpha$-particles have been proven to be potent therapeutic effectors when directed at cancer targets $(11,12)$. The short effective range (several cell diameters) and high energies (5-8 MeV) of these particles make them ideal for targeting the aggressive growing tumor vasculature of glioblastoma while imparting minimal bystander radiation damage to the healthy brain tissue. The therapeutic efficacy of ${ }^{225} \mathrm{Ac}-\mathrm{E} 4 \mathrm{G} 10$ was significant, improving survival versus vehicle-treated controls; ${ }^{225} \mathrm{Ac}-\mathrm{E} 4 \mathrm{G} 10$ increased survival from 9 to $21 \mathrm{~d}$. MRI measurements comparing the tumor volumes of
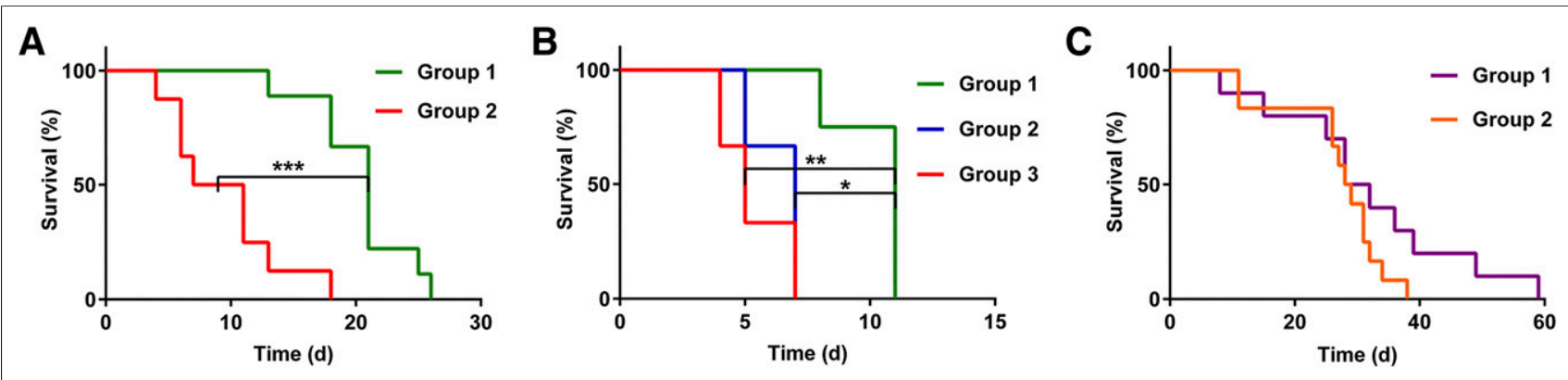

FIGURE 5. Survival studies in Ntva mice with glioblastoma. (A) Kaplan-Meier survival curves of survival study I comparing overall survival of ${ }^{225} \mathrm{Ac}-$ E4G10-treated (group 1) versus vehicle-treated (group 2) Ntva mice. (B) Survival study II showing overall survival after ${ }^{225}$ Ac-E4G10 (group 1) therapy compared with unspecific therapy with ${ }^{225} \mathrm{Ac}$-isotype control antibody (group 2) and vehicle-treated controls (group 3). Animals in survival study II exhibited advanced symptoms of disease. (C) Survival study III comparing overall survival of mice treated with combinational therapy with ${ }^{225} \mathrm{Ac}-$ E4G10 radioimmunotherapy and temozolomide chemotherapy (group 1) versus temozolomide-only (group 2 ). ${ }^{\star} P<0.05 .{ }^{\star \star} P<0.01$. ${ }^{\star \star \star} P<0.001$. 
vehicle-treated versus ${ }^{225} \mathrm{Ac}-\mathrm{E} 4 \mathrm{G} 10$ showed potent tumor growth control. Treatment efficacy and specificity of ${ }^{225}$ Ac-E4G10 was demonstrated in animals with advanced disease (hydrocephalus and hunched posture) in a survival study versus ${ }^{225} \mathrm{Ac}$-isotype and vehicle controls. The combination of ${ }^{225} \mathrm{Ac}-\mathrm{E} 4 \mathrm{G} 10$ and temozolomide increased median survival of glioblastoma mice further to 30 versus $21 \mathrm{~d}$. There was no significant survival difference for the combination therapy compared with temozolomide only; however, we did observe a long-tailed survival curve, indicating a survival benefit in mice treated with radioimmunotherapy plus chemotherapy. Moreover, the hazard ratio showed a survival benefit in the combination group. Temozolomide is currently the chemotherapeutic of choice for the treatment of human glioblastoma; it is a cytotoxic prodrug that methylates nucleotide bases and inhibits DNA replication after hydrolysis (27). Improving temozolomide delivery to glioblastoma as a result of vascular remodeling would be an advantage in this drug's use.

${ }^{225}$ Ac-E4G10-treated animals in survival studies eventually progressed and succumbed to the disease. However, we conducted our $\alpha$-particle therapy studies with a single dose of ${ }^{225} \mathrm{Ac}-\mathrm{E} 4 \mathrm{G} 10$ to provide therapeutic proof of concept and to demonstrate feasibility and efficacy of antivascular therapy with ${ }^{225}$ Ac-E4G10 in glioblastoma mice. Multiple ${ }^{225}$ Ac-E4G10 treatments and optimization of drug scheduling of combined therapies could be used to maximize therapeutic effect.

The pharmacokinetic distribution of ${ }^{225} \mathrm{Ac}-\mathrm{E} 4 \mathrm{G} 10$ in the Ntva mouse model showed drug accumulation in tumor and bone marrow versus the isotype control $10 \mathrm{~d}$ after administration. Both tissues express monomeric VE-cadherin and are therefore sinks for ${ }^{225}$ Ac-E4G10 accumulation. The correlative data from naïve animals support this result. We found moreover a 7.7-fold increase in absorbed dose of ${ }^{225} \mathrm{Ac}-\mathrm{E} 4 \mathrm{G} 10$ to glioblastoma versus healthy brain tissue in naïve mice, explaining efficacy of the radioimmunoconstruct in tumor tissue. Histologic analysis of ${ }^{225}$ Ac-E4G10-treated glioblastoma brain tissue showed an overall vascular depletion and a remodeled microenvironment. We observed a $64 \%$ drop in overall vascularity; vessels were smaller in size and better distributed over the whole tissue.

Estimates of absorbed doses to healthy tissues are crucial for toxicity estimates when treating with $\alpha$-particles (28). We found greater than 20 percent of the injected dose per gram of tissue weight of ${ }^{225} \mathrm{Ac}-\mathrm{E} 4 \mathrm{G} 10$ in the liver $10 \mathrm{~d}$ after treatment, and the estimated dose to liver was the highest among nontargeted tissues (1,577 Gy/MBq in glioblastoma animals). However, similar or even higher doses to liver have been observed with the $\alpha$-emitter ${ }^{213} \mathrm{Bi}$ in leukemia patients treated with ${ }^{213} \mathrm{Bi}$-lintuzumab (29), and no liver toxicity was noted in this study or the ${ }^{225} \mathrm{Ac}$-lintuzumab clinical trials $(30)$.

The concurrent processes of vasculogenesis, angiogenesis, and tumor proliferation are keys to tumor growth and dissemination and interrelated by a paracrine effect. Disrupting and damaging the vascular endothelial architecture associated with tumor tissue is a recognized therapeutic strategy. This approach has been used clinically, with the approval of several antiangiogenic agents by the Food and Drug Administration. However, systemic toxicity and therapy resistance have been important issues when treating with bevacizumab and other antiangiogenic agents. Importantly, our drug acts primarily through direct cell killing, rather than through action on signaling networks and without the global side effects on the angiogenesis axis that other antiangiogenic drugs may promote. Furthermore, targeting endothelial cells in the inner lumen of the glioblastoma vasculature with this macromolecular radioimmunotherapeutic agent permits systemic administration and, importantly, does not require transit across the blood-brain barrier and penetration into the tumor. The requirement to cross the blood-brain barrier and accumulate in tumor could be a major hurdle for other glioblastoma-targeting agents.

\section{CONCLUSION}

Our preclinical studies in the Ntva mouse model found a promising potential of ${ }^{225} \mathrm{Ac}-\mathrm{E} 4 \mathrm{G} 10$ therapy as antivascular treatment for glioblastoma. We demonstrated in survival studies that radioimmunotherapy with ${ }^{225} \mathrm{Ac}-\mathrm{E} 4 \mathrm{G} 10$ in Ntva mice increased overall survival as standalone therapy from 9 to $21 \mathrm{~d}$ and controlled tumor growth. Survival was further increased to $30 \mathrm{~d}$ if the treatment was applied in combination with the standard-of-care chemotherapeutic temozolomide. We showed for the first time how an $\alpha$-targeting antivascular drug in a relevant transgenic mouse model of glioblastoma improved survival. In view of future clinical development of ${ }^{225}$ Ac-E4G10 as antivascular treatment for the therapy of glioblastoma, further studies investigating the mechanism of action of ${ }^{225} \mathrm{Ac}-\mathrm{E} 4 \mathrm{G} 10$ in the Ntva mouse model are necessary. We are currently investigating the changes in the architecture of the vasculature and tumor microenvironment caused by $\alpha$-particle radiation in Ntva mice. Mechanistic studies will inform on the observed survival benefit on radioimmunotherapy reported herein using the ${ }^{225}$ Ac-E4G10 drug in the glioblastoma Ntva mouse model.

\section{DISCLOSURE}

The costs of publication of this article were defrayed in part by the payment of page charges. Therefore, and solely to indicate this fact, this article is hereby marked "advertisement" in accordance with 18 USC section 1734 . This work was supported by the NIH R01 CA166078, R01 CA55349, R25T CA046945, R24 CA83084, P30 CA08748, P01 CA33049, and F31 CA167863; the MSK Experimental Brain Tumor Center; Mr. William H. and Mrs. Alice Goodwin and the Commonwealth Foundation for Cancer Research; The MSK Center for Experimental Therapeutics; and the MSK Center for Molecular Imaging and Nanotechnology. Michael R. McDevitt and David A. Scheinberg declare associations with Actinium Pharmaceuticals, Inc. No other potential conflict of interest relevant to this article was reported.

\section{ACKNOWLEDGMENTS}

We thank Eli Lilly and Company for the E4G10 antibody, Pharmactinium, Inc., for ${ }^{225} \mathrm{Ac}$, Dr. Eric Holland and Dr. Johanna Joyce for breeding pairs and the MSKCC Molecular Cytology Core Facility.

\section{REFERENCES}

1. Dunn GP, Rinne ML, Wykosky J, et al. Emerging insights into the molecular and cellular basis of glioblastoma. Genes Dev. 2012;26:756-784.

2. Chinot OL, Wick W, Mason W, et al. Bevacizumab plus radiotherapy-temozolomide for newly diagnosed glioblastoma. N Engl J Med. 2014;370:709-722.

3. Norden AD, Wen PY. Glioma therapy in adults. Neurologist. 2006;12:279-292.

4. Tuettenberg J, Friedel C, Vajkoczy P. Angiogenesis in malignant glioma: a target for antitumor therapy? Crit Rev Oncol Hematol. 2006;59:181-193.

5. Jain RK, di Tomaso E, Duda DG, Loeffler JS, Sorensen AG, Batchelor TT. Angiogenesis in brain tumours. Nat Rev Neurosci. 2007;8:610-622. 
6. Cloughesy TF, Cavenee WK, Mischel PS. Glioblastoma: from molecular pathology to targeted treatment. Annu Rev Pathol. 2014;9:1-25.

7. Ranpura V, Hapani S, Wu S. Treatment-related mortality with bevacizumab in cancer patients: a meta-analysis. JAMA. 2011;305:487-494.

8. Bergers G, Hanahan D. Modes of resistance to anti-angiogenic therapy. Nat Rev Cancer. 2008;8:592-603.

9. Kerbel RS. Antiangiogenic therapy: a universal chemosensitization strategy for cancer? Science. 2006;312:1171-1175.

10. Jain RK. Normalization of tumor vasculature: an emerging concept in antiangiogenic therapy. Science. 2005;307:58-62.

11. McDevitt MR, Sgouros G, Finn RD, et al. Radioimmunotherapy with alphaemitting nuclides. Eur J Nucl Med. 1998;25:1341-1351.

12. McDevitt MR, Ma D, Lai LT, et al. Tumor therapy with targeted atomic nanogenerators. Science. 2001;294:1537-1540.

13. Sgouros G, Hobbs RF, Song H. Modelling and dosimetry for alpha-particle therapy. Curr Radiopharm. 2011;4:261-265.

14. FDA approves radiopharmaceutical for metastatic prostate cancer. Cancer Discov. 2013;3:OF1.

15. Sgouros G, Roeske JC, McDevitt MR, et al. MIRD pamphlet no. 22 (abridged): radiobiology and dosimetry of $\alpha$-particle emitters for targeted radionuclide therapy. J Nucl Med. 2010;51:311-328.

16. Liao F, Doody JF, Overholser J, et al. Selective targeting of angiogenic tumor vasculature by vascular endothelial-cadherin antibody inhibits tumor growth without affecting vascular permeability. Cancer Res. 2002;62:2567-2575.

17. May C, Doody JF, Abdullah R, et al. Identification of a transiently exposed VEcadherin epitope that allows for specific targeting of an antibody to the tumor neovasculature. Blood. 2005;105:4337-4344.

18. Nolan DJ, Ciarrocchi A, Mellick AS, et al. Bone marrow-derived endothelial progenitor cells are a major determinant of nascent tumor neovascularization. Genes Dev. 2007;21:1546-1558.
19. Maguire WF, McDevitt MR, Smith-Jones PM, Scheinberg DA. Efficient 1-step radiolabeling of monoclonal antibodies to high specific activity with ${ }^{225}$ Ac for $\alpha$-particle radioimmunotherapy of cancer. J Nucl Med. 2014;55:1492-1498.

20. McDevitt MR, Ma D, Simon J, Frank RK, Scheinberg DA. Design and synthesis of ${ }^{225}$ Ac radioimmunopharmaceuticals. Appl Radiat Isot. 2002;57:841-847.

21. Hambardzumyan D, Amankulor NM, Helmy KY, Becher OJ, Holland EC. Modeling adult gliomas using RCAS/t-va technology. Transl Oncol. 2009;2:89-95.

22. Guide for the Care and Use of Laboratory Animals. Bethesda, MD: National Institutes of Health; 1985. NIH publication 85-23.

23. Escorcia FE, Henke E, McDevitt MR, et al. Selective killing of tumor neovasculature paradoxically improves chemotherapy delivery to tumors. Cancer Res. 2010;70:9277-9286.

24. Ruggiero A, Villa CH, Holland JP, et al. Imaging and treating tumor vasculature with targeted radiolabeled carbon nanotubes. Int J Nanomedicine. 2010;5:783-802.

25. Singh Jaggi J, Henke E, Seshan SV, et al. Selective alpha-particle mediated depletion of tumor vasculature with vascular normalization. PLoS One. 2007; 2:e267.

26. Huse JT, Holland EC. Genetically engineered mouse models of brain cancer and the promise of preclinical testing. Brain Pathol. 2009;19:132-143.

27. Weller M, Cloughesy T, Perry JR, Wick W. Standards of care for treatment of recurrent glioblastoma-are we there yet? Neuro-oncol. 2013;15:4-27.

28. Akabani G, McLendon RE, Bigner DD, Zalutsky MR. Vascular targeted endoradiotherapy of tumors using $\alpha$-particle-emitting compounds: theoretical analysis. Int J Radiat Oncol Biol Phys. 2002;54:1259-1275.

29. Sgouros G, Ballangrud AM, Jurcic JG, et al. Pharmacokinetics and dosimetry of an alpha-particle emitter labeled antibody: ${ }^{213} \mathrm{Bi}-\mathrm{HuM} 195$ (anti-CD33) in patients with leukemia. $J$ Nucl Med. 1999;40:1935-1946.

30. Sgouros G, ed. MIRD Radiobiology and Dosimetry for Radiopharmaceutical Therapy with Alpha-Particle Emitters. Reston, VA: Society of Nuclear Medicine and Molecular Imaging, Inc.; 2015. 